\title{
Microbial Colonization of Pneumatic Tourniquets in the Orthopedic Operating Room
}

Syed H. Mufarrih ${ }^{1}$, Nada Q. Qureshi ${ }^{2}$, Rizwan H. Rashid ${ }^{1}$, Bilal Ahmed ${ }^{3}$, Seema Irfan ${ }^{4}$, Akbar J. Zubairi ${ }^{1}$, Shahryar Noordin ${ }^{1}$

1. Orthopedic Surgery, Aga Khan University, Karachi, PAK 2. Cardiology, Aga Khan University, Karachi, PAK 3. Medicine, Aga Khan University, Karachi, PAK 4. Microbiology, Aga Khan University, Karachi, PAK

$\square$ Corresponding author: Syed H. Mufarrih, hamzamufarrih@live.com Disclosures can be found in Additional Information at the end of the article

\section{Abstract}

\section{Background}

The rate of surgical site infections following orthopedic procedures is approximately $2 \%$ globally. Potential sources of contamination in the operating room include pneumatic tourniquets, blood pressure cuffs, and stethoscopes, among others. Our study aims to investigate microbial colonization on reusable pneumatic tourniquets stored and used in the orthopedic department of our institution and evaluate the efficacy of the cleaning protocols employed.

\section{Methods}

Over a course of two weeks, 26 samples were obtained. A total of 14 pneumatic tourniquets were sampled preoperatively on Monday morning following the weekly cleaning protocol of soaking the tourniquets in sodium hypochlorite for 30 minutes while 12 tourniquets were cultured immediately following the postoperative cleaning protocol of wiping the tourniquet clean with a cloth soaked in sodium hypochlorite. Samples were cultured on MacConkey and sheep blood agar and incubated at 37-degrees centigrade for a total of 48 hours. Organisms were identified and colony count was documented. The analysis was performed using the Fisher Exact test on SPSS v23 (IBM Corp., Armonk, NY, US).

\section{Results}

All 14 samples obtained after being soaked in sodium hypochlorite for 30 minutes cultured negative. However, four out of 12 (33\%) samples obtained after simply wiping the pneumatic tourniquet with a cloth soaked in sodium hypochlorite cultured coagulase-negative

Received 07/03/2019 Review began 07/29/2019 Review ended 07/31/2019 Published 08/02/2019

\section{(C) Copyright 2019}

Mufarrih et al. This is an open access article distributed under the terms of the Creative Commons Attribution License CC-BY 3.0., which permits unrestricted use, distribution, and reproduction in any medium, provided the original author and source are credited.
Staphylococci. The difference between the two was significant $(\mathrm{p}=0.002)$.

\section{Conclusion}

Postoperative tourniquets, wiped with a cloth soaked in sodium hypochlorite and ready to be used on the next patient, were found to be contaminated with coagulase-negative Staphylococcus. This species is notorious for causing surgical site infections following implantrelated surgeries potentially through direct inoculation and cross-infections intraoperatively and in storage. Efforts to identify the relationship with postoperative surgical site infections need to be made to suggest more aggressive cleaning protocols. 
Categories: Infectious Disease, Orthopedics

Keywords: tourniquet, surgical site infection, joint arthroplasty, operating room, infection

\section{Introduction}

Surgical site infections (SSIs) are an unfortunate complication of extremity surgery, even more so where implants are involved such as in fracture fixation and joint arthroplasty. Besides the significant morbidity associated with surgical site infections, the economic implications are huge owing to the increase in the length of hospital stay, need for revision surgery, and multiple hospitalizations, collectively contributing to an approximately $300 \%$ increase in the cost of surgery [1]. Given the current SSI rate of approximately 2\% [2-3] following orthopedic procedures and the potentially preventable nature of the complication, it is imperative to direct all efforts to tackle this issue head-on.

Previous literature has identified stethoscopes [4-6], mobile phones [5], marking pens [7], and blood-pressure cuffs [7] as possible sources of infection in the operating room.

The reusable pneumatic tourniquet, a "non-critical” item as per the Spaulding classification, has been highlighted as a possible source of infection following orthopedic procedures [8]. Pneumatic tourniquets are employed in orthopedic procedures to maintain a bloodless field for the duration of the surgery. Previous studies have shown that many tertiary care hospitals lack a standard protocol for the cleaning of the tourniquets [9], and the same tourniquet may be used on patients with both clean and contaminated wounds. Subsequently, contaminated tourniquets may be stored with the other tourniquets without adequate disinfection. Hence, we hypothesized that pneumatic tourniquets maybe serve as a potential reservoir for pathogenic bacteria.

The aim of our study was to investigate whether reusable pneumatic tourniquets are colonized by microbes implicated in postoperative surgical site infections following orthopedic procedures and evaluate the efficacy of the pre and postoperative cleaning protocols employed at our institution.

\section{Materials And Methods}

\section{Study design}

A cross-sectional study was conducted at the orthopedic operating rooms of Aga Khan University Hospital (AKUH), Karachi, from August 2017 to September 2017. Preoperative and postoperative samples were collected from the 14 pneumatic tourniquets stored in the orthopedic operating rooms' storage unit. The purpose of this study was to investigate the microbial colonization on pneumatic tourniquets employed in extremity surgery.

\section{Sampling}

Materials

At our university hospital, 14 reusable pneumatic tourniquets are stored together in a pneumatic box for use in the orthopedic department exclusively. The available tourniquets had a range of sizes, with an outer fabric layer with velcro and an inner silicone bladder. An inflatable balloon made of latex covered the entire length. These tourniquets are used in most orthopedic surgeries in close proximity to the surgical site throughout the length of the procedure.

Timings of Pre and Postoperative Sampling 
Two types of cleaning protocols are employed at our institution. Once weekly, all tourniquets are soaked in sodium hypochlorite for 30 minutes. Between surgeries, after being used on one patient before moving on to the next, the tourniquet is simply wiped with a cloth soaked in sodium chlorite. We obtained "preoperative" samples following the once-weekly cleaning protocol on Monday morning and "postoperative” samples following the wiping of the tourniquet with sodium hypochlorite. This was done to assess the adequacy of each protocol. Tourniquets employed in surgical debridement were not used in our study, as they are classified as “dirty surgeries.” During the course of sampling, two tourniquets at the extremes of sizes were not employed in any procedure and, therefore, their postoperative sample was not obtained.

\section{Procedure}

In collaboration with the microbiology department of our institute, a standardized protocol for sample collection from each tourniquet was developed. An orthopedic resident, trained in the sample collection technique by the microbiology department was responsible for obtaining all samples. Necessary measures to prevent cross-infection, such as the use of gloves and a mask, were taken during sampling. A single swab on a stick soaked in normal saline was used to wipe a one-centimeter square area from five points on the posterior surface (which has contact with the patient skin) of each tourniquet. Four points were each at a distance of one centimeter from the four edges, with the fifth one at the center (Figure 1). The swab was then sealed in a test tube containing normal saline and was sent for culture to the microbiology department and processed within 24 hours without any storage phase.

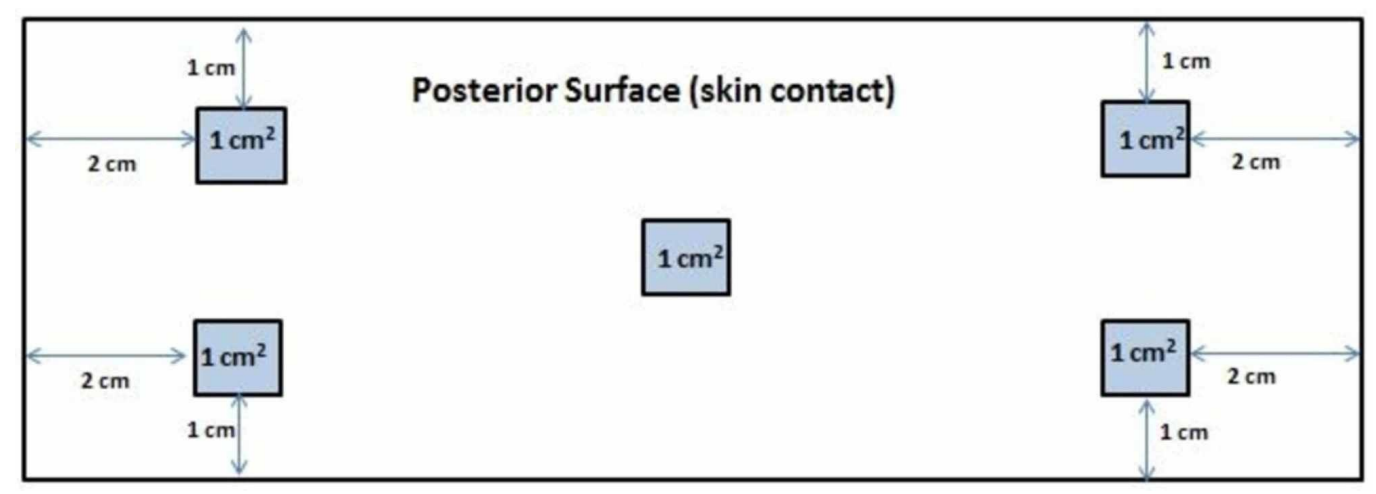

FIGURE 1: Technique for obtaining sample swabs from pneumatic tourniquets.

\section{Laboratory procedure}

The sample swabs were cultured on both MacConkey and sheep blood agar and incubated at 37degree centigrade for 24 hours. If no growth was seen, the culture plate was reincubated for an additional 24 hours. In cases of bacterial growth, the colony-forming units (CFUs) on the culture plate were counted. Finally, culture mediums were tested for the growth of Staphylococcus species, including Staphylococcus aureus (MSSA/MRSA) and coagulasenegative Staphylococcus (CoNS), Pseudomonas species, Acinetobacter species, Streptococcus species, and Klebsiella species. Growth on the culture media was documented as the bacterial species with colony-forming units.

\section{Statistical analysis}




\section{Cureus}

The results of preoperative and postoperative cultures from all tourniquets have been tabulated. Data were analyzed using SPSS version 22 (IBM Corp., Armonk, NY, US). Simple frequencies and proportions were calculated to describe categorical data. To calculate the $95 \%$ confidence intervals (CIs) for the proportion of tourniquets getting contaminated postoperatively, the Fisher Exact test was used.

\section{Results}

All 14 preoperative samples did not culture any microorganisms. Out of the 12 postoperative samples, four (33.3\%) were contaminated. Those that were contaminated had a colony count between one and two colony-forming units. Coagulase-negative Staphylococcus was the only organism isolated (Table 1). The difference between preoperative and postoperative contamination was significant. The postoperative decontamination protocol of wiping the tourniquet with a cloth soaked in sodium hypochlorite is not effective when compared to the preoperative decontamination protocol of soaking the tourniquet in the sodium hypochlorite solution $(\mathrm{p}=0.002)$.

\begin{tabular}{|c|c|c|c|}
\hline Tourniquet & Pre-operative & Post-operative & Colony Forming Units (CFUs) \\
\hline 1 & Not isolated & Not Isolated & \\
\hline 2 & Not isolated & Sample not sent & - \\
\hline 3 & Not isolated & Not isolated & - \\
\hline 4 & Not isolated & Coagulase-negative Staphylococcus & 1 \\
\hline 5 & Not isolated & Not isolated & - \\
\hline 6 & Not isolated & Not isolated & - \\
\hline 7 & Not isolated & Coagulase-negative Staphylococcus & 1 \\
\hline 8 & Not isolated & Sample not sent & - \\
\hline 9 & Not isolated & Not isolated & - \\
\hline 10 & Not isolated & Coagulase-negative Staphylococcus & 2 \\
\hline 11 & Not isolated & Not isolated & - \\
\hline 12 & Not isolated & Coagulase-negative Staphylococcus isolated & 2 \\
\hline 13 & Not isolated & Not isolated & - \\
\hline 14 & Not isolated & Not isolated & - \\
\hline
\end{tabular}

TABLE 1: Qualitative and quantitative results of growth on culture media.

\section{Discussion}

With the reported increase in life expectancy by the World Health Organization (WHO), osteoarthritis is expected to become the fourth leading cause of disability by 2020 [10]. Needless 
to say, the incidence of arthroplasty, which is the gold standard for the treatment of end-stage osteoarthritis, is expected to rise significantly $[2,11]$. According to available literature, the incidence of postoperative surgical site infections for joint arthroplasty is approximately $2 \%$ [12-15]. These statistics are alarming when the devastating consequences of an SSI, including increased length of hospital stay, with more than double the hospital costs and higher complication and mortality rates [13], are taken into account.

Despite the advancement of medical science, the war against infection continues to persist. Pneumatic tourniquets, classified as "non-critical" items by the Spaulding classification, have been implicated as a source of contamination in the operating room [16]. Changing the resistance patterns of microbes and accountability for the biofilm activity of the organisms may push for items, including tourniquets, stethoscopes, and marking pens, among others, to demand more aggressive cleaning protocols [17-19].

The use of pneumatic tourniquets in joint arthroplasty is now predominantly part of the standard procedure, as it benefits surgeons by maintaining a bloodless field, improving the visualization of important structures, strengthening the bone cement interface, and expediting the procedure [20]. However, recent studies have shown that reusable tourniquets are indeed contaminated and have postulated their potential role in the development of surgical site infections [8,21-22].

Compared to previous studies, which have reported contamination of up to $80 \%$ of the tested tourniquets with much higher colony counts, contamination in our study is low [8,23-24]. An obvious reason is that our study intended to investigate the efficacy of cleaning protocols and thus obtained samples after some cleaning mechanism had been employed. Other possible contributors for this difference may be the exclusion of cases of surgical debridement in our study and the inclusion of trauma-related orthopedic procedures in the referenced study.

Coagulase-negative Staphylococcus was the only organism isolated from our tourniquets. Although a part of the normal flora of the skin and mucous membranes, CoNS is among the most common causes of surgical site infections in orthopedic surgery along with Staphylococcus aureus, Enterococci, and Streptococci [24-27]. They are now gaining importance as nosocomial pathogens due to the emergence of methicillin-resistant strains, which may act as reservoirs for genetic material, particularly SCCmec IVa, found in MRSA [28-29].

Isolating this notorious species from tourniquets that were ready to be used in the next procedure is alarming, as the contaminated tourniquets may be a source of surgical site infections through various mechanisms. Firstly, direct inoculation of the surgical site by the bacteria on the tourniquet can occur through staff who fail to follow a proper hand hygiene protocol due to the non-critical status of the pneumatic tourniquet [30]. The use of a contaminated tourniquet can go on to serve as a source of infection in all subsequent surgeries until the standard weekend decontamination is performed. This phenomenon of crossinfection has been reported in previous studies as well [8,24]. Furthermore, the same tourniquet when stored in a pneumatic box with the rest may contaminate the other tourniquets, particularly if the scenario involves use in a traumatic or wound debridement surgery.

To decrease the rate of surgical site infections, efforts should be made to establish the relationship of colonization to surgical site infections, explore the contamination of other items in the operating room, such as marking pens, blood pressure cuffs, and stethoscopes, and investigate the efficacy of cost-effective cleaning methods.

The findings of our study are based on the cultures taken from a single center. To draw 
definitive conclusions, the study needs to be replicated in other centers. Multiple batches may be tested if the number of tourniquets available is small. Furthermore, in order to establish a causal relationship between colonization and infection, methods for the typing of bacterial strains, such as pulse-field gel electrophoresis, need to be utilized.

\section{Conclusions}

Re-usable pneumatic tourniquets cleaned by wiping with a cloth soaked in sodium hypochlorite may still be contaminated with potentially pathogenic bacteria when compared to soaking the tourniquet in sodium hypochlorite for 30 minutes. The colonization of pneumatic tourniquets may contribute to surgical site infections following orthopedic procedures in several ways, including direct inoculation, breaching of hand hygiene protocol, and cross-infection. Efforts to establish a causal relationship between contamination and surgical site infections need to be made before suggesting more aggressive cleaning protocols.

\section{Additional Information}

\section{Disclosures}

Human subjects: All authors have confirmed that this study did not involve human participants or tissue. Animal subjects: All authors have confirmed that this study did not involve animal subjects or tissue. Conflicts of interest: In compliance with the ICMJE uniform disclosure form, all authors declare the following: Payment/services info: All authors have declared that no financial support was received from any organization for the submitted work. Financial relationships: All authors have declared that they have no financial relationships at present or within the previous three years with any organizations that might have an interest in the submitted work. Other relationships: All authors have declared that there are no other relationships or activities that could appear to have influenced the submitted work.

\section{References}

1. Whitehouse JD, Friedman ND, Kirkland KB, Richardson WJ, Sexton DJ: The impact of surgicalsite infections following orthopedic surgery at a community hospital and a university hospital adverse quality of life, excess length of stay, and extra cost. Control Hosp Epidemiol. 2002, 23:183-189. 10.1086/502033

2. Singh JA: Epidemiology of knee and hip arthroplasty: a systematic review . Open Orthop J. 2011, 5:80-85. 10.2174/1874325001105010080

3. Badawy M: Influence of hospital procedure volume on the risk of revision in knee arthroplasty surgery. University of Bergen, AIT Bjerch AS, Norway; 2016.

4. Marinella MA, Pierson C, Chenoweth C: The stethoscope: a potential source of nosocomial infection?. Arch Intern Med. 1997, 157:786-790. 10.1001/archinte.1997.00440280114010

5. Kennedy KJ, Dreimanis DE, Beckingham WD, Bowden FJ: Staphylococcus aureus and stethoscopes. Med J Aust. 2003, 178:468.

6. Datta P, Bansal N, Chander J: Hand-held hazards by health-care workers. Indian J Med Microbiol. 2013, 31:320-321. 10.4103/0255-0857.115675

7. French G, Rayner D, Branson M, Walsh M: Contamination of doctors' and nurses' pens with nosocomial pathogens. Lancet. 1998, 351:213. 10.1016/S0140-6736(05)78182-4

8. Ahmed S, Ahmad R, Case R, Spencer R: A study of microbial colonisation of orthopaedic tourniquets. Ann R Coll Surg Engl. 2009, 91:131-134. 10.1308/003588409X359402

9. Ben-David D, Walsh EF, Ritter M, Mechrefe AP, Mermel LA, DiGiovanni CW: Microbial colonization of tourniquets used in orthopedic surgery. Orthopedics. 2006, 29:709-713.

10. Woolf AD, Pfleger B: Burden of major musculoskeletal conditions. Bull World Health Organ. 2003, 81:646-656.

11. Issa K, Rifai A, Boylan MR, Pourtaheri S, McInerney VK, Mont MA: Do various factors affect the frequency of manipulation under anesthesia after primary total knee arthroplasty?. Clin Orthop Relat Res. 2015, 473:143-147. 10.1007/s11999-014-3772-x

12. Urquhart DM, Hanna FS, Brennan SL, et al.: Incidence and risk factors for deep surgical site 
infection after primary total hip arthroplasty: a systematic review. J Arthroplasty. 2010, 25:1216-1222. 10.1016/j.arth.2009.08.011

13. Minnema B, Vearncombe M, Augustin A, Gollish J, Simor AE: Risk factors for surgical-site infection following primary total knee arthroplasty. Infect Control Hosp Epidemiol. 2004, $25: 477-480.10 .1086 / 502425$

14. Mufarrih SH, Aqueel T, Ali A, Malik AT, Noordin S: Unilateral vs. bilateral total knee arthroplasty with 90-day morbidity and mortality: a retrospective cohort study. Int J Surg. 2017, 8:24-28. 10.1016/j.ijso.2017.06.003

15. Wilson J, Charlett A, Leong G, McDougall C, Duckworth G: Rates of surgical site infection after hip replacement as a hospital performance indicator: analysis of data from the English mandatory surveillance system. Infect Control Hosp Epidemiol. 2008, 29:219-226. $10.1086 / 527511$

16. BC Ministry of Health, BC Health Authorities, Providence Health Care: Best Practice Guidelines for Cleaning, Disinfection and Sterilization of Critical and Semi-critical Medical. BC Ministry of Health, Canada; 2011.

17. Lewis T, Patel V, Ismail A, Fraise A: Sterilisation, disinfection and cleaning of theatre equipment: do we need to extend the Spaulding classification?. J Hosp Infect. 2009, 72:361363. 10.1016/j.jhin.2009.04.008

18. McDonnell G, Burke P: Disinfection: is it time to reconsider Spaulding? . J Hosp Infect. 2011, 78:163-170. 10.1016/j.jhin.2011.05.002

19. Tiwari S, Rajak S, Mondal DP, Biswas D: Sodium hypochlorite is more effective than $70 \%$ ethanol against biofilms of clinical isolates of Staphylococcus aureus. Am J Infect Control. 2018, 46:e37-e42. 10.1016/j.ajic.2017.12.015

20. Estebe J-P, Davies JM, Richebe P: The pneumatic tourniquet: mechanical, ischaemiareperfusion and systemic effects. Eur J Anaesthesiol. 2011, 28:404-411.

10.1097/EJA.0b013e328346d5a9

21. Thompson S, Middleton M, Farook M, Cameron-Smith A, Bone S, Hassan A: The effect of sterile versus non-sterile tourniquets on microbiological colonisation in lower limb surgery. Ann R Coll Surg Engl. 2011, 93:589-590. 10.1308/147870811X13137608455334

22. Yi S, Tan J, Chen C, Chen H, Huang W: The use of pneumatic tourniquet in total knee arthroplasty: a meta-analysis. Arch Orthop Trauma Surg. 2014, 134:1469-1476.

10.1007/s00402-014-2056-y

23. Golder M, Chan C, O'Shea S, Corbett K, Chrystie I, French G: Potential risk of cross-infection during peripheral-venous access by contamination of tourniquets. Lancet. 2000, 355:44. 10.1016/s0140-6736(99)04051-9

24. Brennan SA, Walls RJ, Smyth E, Mulla TA, O'Byrne JM: Tourniquets and exsanguinators: a potential source of infection in the orthopedic operating theater?. Acta Orthopaedica. 2009, 80:251-255. 10.3109/17453670902930016

25. Pulido L, Ghanem E, Joshi A, Purtill JJ, Parvizi J: Periprosthetic joint infection: the incidence, timing, and predisposing factors. Clin Orthop Relat Res. 2008, 466:1710-1715.

10.1007/s11999-008-0209-4

26. Phillips JE, Crane T, Noy M, Elliott T, Grimer RJ: The incidence of deep prosthetic infections in a specialist orthopaedic hospital: a 15-year prospective survey. J Bone Joint Surg Br. 2006, 88:943-948. 10.1302/0301-620X.88B7.17150

27. Owens C, Stoessel K: Surgical site infections: epidemiology, microbiology and prevention. J Hosp Infect. 2008, 70:3-10. 10.1016/S0195-6701(08)60017-1

28. Barbier F, Ruppé E, Hernandez D, et al.: Methicillin-resistant coagulase-negative staphylococci in the community: high homology of SCCmec IVa between Staphylococcus epidermidis and major clones of methicillin-resistant Staphylococcus aureus. J Infect Dis. 2010, 202:270-281. 10.1086/653483

29. Eady EA, Cove JH: Staphylococcal resistance revisited: community-acquired methicillin resistant Staphylococcus aureus - an emerging problem for the management of skin and soft tissue infections. Curr Opin Infect Dis. 2003, 16:103-124.

10.1097/01.aco.0000065071.06965.ca

30. Pittet D, Mourouga P, Perneger TV: Compliance with handwashing in a teaching hospital . Ann Intern Med. 1999, 130:126-130. 10.7326/0003-4819-130-2-199901190-00006 